\title{
Maternal and Hospital Factors Associated with First-Time Mothers' Breastfeeding Practice: A Prospective Study
}

\author{
Tzu-I Tsai, ${ }^{1}$ Shu-Her Huang, ${ }^{1}$ and Shoou-Yih D. Lee ${ }^{2}$
}

\begin{abstract}
Continuity of breastfeeding is infrequent and indeterminate. Evidence is lacking regarding factors associated with breastfeeding at different postpartum time points. This prospective study investigated the change in, and correlates of, breastfeeding practices after delivery at a hospital and at 1, 3, and 6 months postpartum among first-time mothers. We followed a cohort of 300 primiparous mothers of Taiwan who gave birth at two hospitals during 2010-2011. Logistic and Cox regression analyses were performed to determine factors that were correlated with breastfeeding practices. In the study sample, the rate of exclusive breastfeeding during the hospital stay was $66 \%$; it declined to $37.5 \%$ at 1 month and $30.2 \%$ at 3 months postpartum. Only $17.1 \%$ of women reported continuing breastfeeding at 6 months. Early initiation of breastfeeding, rooming-in practice, and self-efficacy were significantly related to exclusive breastfeeding during the hospital stay. After discharge, health literacy, knowledge, intention, and self-efficacy were positively and significantly associated with breastfeeding exclusivity. Later initiation (hazard ratio $=1.53 ; 95 \%$ confidence interval, 1.05, 1.97), shorter intention (hazard ratio $=1.42 ; 95 \%$ confidence interval, 1.13, 1.68), and self-efficacy (hazard ratio $=0.98 ; 95 \%$ confidence interval, 0.96, 0.99) were important predictors of breastfeeding cessation within 6 months of delivery. Continuous breastfeeding practice for 6 months is challenging and difficult for new mothers. Results showed that factors related to breastfeeding varied over time after delivery. Interventions seeking to sustain breastfeeding should consider new mothers' needs and barriers at different times.
\end{abstract}

\section{Introduction}

B REASTFEEDING has Numerous NUTRITIONAL, developmental, psychological, immunological, social, economic, and environmental benefits. ${ }^{1,2}$ The World Health Organization (WHO) advocates for timely initiation of breastfeeding and exclusive breastfeeding up to 6 months after delivery. ${ }^{3}$ Despite research and advocacy efforts, only $34.8 \%$ of infants worldwide are estimated to be exclusively breastfed for the recommended 6 months. The average rate of exclusive breastfeeding within 6 months postpartum among countries in the East Asian and Pacific Region is $30 \% .{ }^{4}$ The breastfeeding rate in Taiwan is even lower. A recent study, the Taiwan Birth Cohort Study, reported a high initiation rate (83.7\%), but the rate of exclusive and partial breastfeeding dropped to $25.4 \%$ at 6 months. ${ }^{5}$

Continuous breastfeeding depends on a constant effort to establish the practice. There is a wealth of literature de- scribing how maternal sociodemographic and psychosocial factors affect infant feeding decisions and practices. Evidence indicates that breastfeeding knowledge, intention, and self-efficacy are positively associated with increased initiation and continuation of breastfeeding. ${ }^{6-9}$ Also important are hospital policies and interventions that promote exclusive breastfeeding. For example, the Baby-Friendly Hospital Initiative (BFHI) proposed by the United Nations Children's Fund (UNICEF) and WHO has been shown to effectively increase the rates of breastfeeding initiation and exclusivity, as well as the duration of breastfeeding. ${ }^{10-12}$ The BFHI recommends a 10-Step Program for successful breastfeeding. Of the 10 Steps, early initiation of breastfeeding, full roomingin, breastfeeding on demand, and exclusive breastfeeding during the hospital stay appear to have a particularly strong and positive influence on breastfeeding duration. ${ }^{11,13,14}$

Notwithstanding an extensive body of research on breastfeeding, we know relatively little about how breastfeeding

\footnotetext{
${ }^{1}$ School of Nursing, National Yang-Ming University, Taipei, Taiwan.

${ }^{2}$ Department of Health Management and Policy, University of Michigan School of Public Health, Ann Arbor, Michigan.

Preliminary results of this study were presented in the 21th International Union of Health Promotion and Education, held in Pattaya, Thailand, August 26-31, 2013.
} 
practices change during the postpartum period and the extent to which factors related to breastfeeding are time specific. With few exceptions, existing research tends to use a crosssectional design and has failed to follow maternal breastfeeding for an extended period. ${ }^{7,15}$ Of the few longitudinal studies, most are focused on either maternal or hospital factors. ${ }^{9,12,16}$ Rarely is there a study that examines systematically how maternal psychosocial attributes and hospital practices influence breastfeeding behaviors over time. ${ }^{8}$

Extant research has also largely ignored the impact of health literacy on breastfeeding practices. An accumulated body of evidence has shown that health literacy — defined as an individual's ability to obtain, process, and understand basic health information and service-affects individual health knowledge, self-efficacy, health resources navigation, health communication, and adherence to healthful behaviors. ${ }^{17-20}$ An exception in breastfeeding research that considered health literacy was Kaufman et al., ${ }^{21}$ who reported that women with a higher health literacy level were more likely to initiate breastfeeding and continue exclusive breastfeeding for the first 2 months after delivery. The study used a cross-sectional design and therefore did not examine the time-varying effect of health literacy on breastfeeding during the postpartum period.

This study had three specific objectives. The first was to examine the change in breastfeeding practices during the postpartum period to answer the question of whether breastfeeding practices sustainable. The second was to examine whether cognitive and psychosocial factors (breastfeeding knowledge, breastfeeding intent, self-efficacy, and health literacy) and inpatient interventions (initiation, roomingin, and prenatal classes) were associated with breastfeeding at different time points after delivery. The third was to examine what maternal and hospital intervention factors were associated with early cessation of breastfeeding within 6 months of delivery. The study results serve to fill the knowledge gap in relation to maternal and hospital determinants for breastfeeding practices and duration during the postpartum period. Identification of those determinants is useful for devising appropriate breastfeeding promotion programs in hospitals and communities.

\section{Materials and Methods}

\section{Data}

We adopted a prospective, longitudinal design and used a survey to collect data from a purposeful sample of women who had their delivery at two hospitals, one located in a metropolitan city (Taipei) and the other located in a suburban area (Yi-Lan), in Taiwan. Both hospitals were BFHIaccredited. (The BFHI was introduced in Taiwan in 2001. By 2012, 163 hospitals [43.8\% of all Taiwanese hospitals, delivering $75.1 \%$ of births nationwide] became BFHI-accredited healthcare institutions, according to the 2013 bureau of health administration (BHA) annual report.)

To be eligible for participation, women had to (1) be primiparous, (2) have no major medical complications or psychological illnesses, and (3) give birth to a healthy, full-term newborn ( $\geq 37$ weeks, weighing $\geq 2,500 \mathrm{~g}$, having an Apgar score of $\geq 8$ at 5 minutes). The sample size (150 at each hospital) was determined based on a power calculation and an estimated attrition rate. With a significance level $\alpha=5 \%$, power $=0.8$, and effect size $=0.25$ and using power calculation software $\left(\mathrm{G}^{*}\right.$ Power version 3.1), we obtained a minimal sample of 120 at each hospital. We estimated the average attrition rate to be $20 \%$ because a previous longitudinal study of breastfeeding mothers experienced $11 \%$ attrition at 3 months and $30 \%$ at 6 months. ${ }^{22}$

The institutional review boards of two institutions approved the protocol. Between 2010 and 2011, a research assistant attended daily the maternity wards to recruit eligible women. The recruitment continued until we reached the targeted sample size. The response rates were $70.4 \%$ at the hospital in Taipei and $78.9 \%$ at the hospital in Yi-Lan. Women who met the eligibility criteria and were willing to participate in the study were asked to sign an informed consent and complete the baseline survey prior to the discharge. The baseline survey collected data on demographic factors, health literacy, breastfeeding knowledge, breastfeeding intent, self-efficacy, breastfeeding and related behaviors (i.e., initiation, rooming-in), and prenatal classes. Follow-up questionnaires were mailed to the participants at 1 month and 3 months postpartum. A final telephone survey was conducted at 6 months. Information collected during follow-up surveys included breastfeeding practices, breastfeeding difficulties, and reasons for discontinuing breastfeeding. The attrition rates were $7.7 \%(n=23)$ at 1 month, $3.2 \%(n=9)$ at 3 months, and $3.7 \%(n=10)$ at 6 months. A total of 258 women completed all four waves of the survey. Participants who missed one of the surveys were not significantly different from those who completed all four surveys in terms of age, education, and residential areas.

\section{Measurements}

"Breastfeeding practice" was assessed by asking respondents the methods of child feeding in the past 24 hours. Following the method of Labbok and Krasovec, ${ }^{23}$ we collected information on breastfeeding practice according to the following categories: exclusive, almost exclusive, high partial, medium partial, low partial, token, and formula only. In the analysis, we combined exclusive and almost exclusive into "exclusive breastfeeding," high partial, medium partial, and low partial into "mixed breastfeeding," and token and formula only into "formula feeding."

"Health literacy" was measured using the Mandarin Health Literacy Scale. ${ }^{24}$ The Mandarin Health Literacy Scale contained 50 items, of which 33 tested comprehension of health-related texts and 17 assessed numeracy skills. Each correct answer is given 1 point, and the total score ranges from 0 to 50, with a higher score indicating a higher level of health literacy. Cronbach's alpha of the Mandarin Health Literacy Scale was 0.95.

Breastfeeding knowledge was measured by 10 survey items that assessed respondents' correct understanding of the benefits and management of breastfeeding (e.g., physiology of breastfeeding, appropriate intervals of breastfeeding, necessary provision of water and other supplements). The items were constructed based on breastfeeding recommendations by UNICEF, the American Academy of Family Physicians, the International Consultant Association, and La Leche League International. Five experts (two obstetrician/ gynecologists and three maternity nurses) evaluated the content validity of the items, rating the relevance and clarity 
of each item on a 4-point Likert scale. The resulting content validity index was 0.92 , indicating good content validity. The respondent received 1 point for each correct answer, so the score ranged from 0 to 10 , with a higher score indicating a higher level of breastfeeding knowledge.

Breastfeeding self-efficacy was assessed using the Chinese version of the Breastfeeding Self-Efficacy Scale. ${ }^{25}$ The Breastfeeding Self-Efficacy Scale consisted of 12 selfreported items on a 5-point Likert scale, with a higher score indicating a higher level of breastfeeding self-efficacy. Cronbach's alpha for the Chinese version of the Breastfeeding Self-Efficacy Scale was 0.93.

"Breastfeeding intent" was assessed by the item "How long do you intend to breastfeed?" and was categorized into "less than 6 months," "about 6 months," and "more than 6 months."

"Birth method" was operationalized as a vaginal delivery or cesarean section.

"Prenatal classes" was assessed by asking participants whether they attended prenatal education classes before giving birth.

"Rooming-in practice" was assessed by asking participants if their baby stayed with them at the bedside.

"Initiation of breastfeeding" was measured based on selfreported timing of the first sucking following delivery, categorized as "within 1 hour after birth," "within 4 hours of birth," "within 24 hours of birth," and "later than 24 hours of birth."

Several demographic characteristics were included as control variables: age, educational attainment, employment status before birth and at 3 months after birth, and residential location.

\section{Statistical analysis}

To address the first objective, we performed descriptive analysis to examine change in the rate of breastfeeding practices. For the second research objective, we performed logistic regression analysis to test the associations of factors with exclusive breastfeeding before discharge and at 1 month and 3 months postpartum, respectively. Finally, to answer the third research question, we used Cox regression analysis to identify factors that were associated with early cessation of breastfeeding within 6 months postpartum. The level of significance was set at $p<0.05$. SPSS version 20 software (SPSS, Inc., Chicago, IL) was used for the descriptive and logistic regression analysis, and SAS software (SAS Institute, Cary, NC) was used for the Cox regression analysis.

\section{Results}

The majority of women in the sample were 25-35 years old, had a college degree or higher level of education, and were employed full-time. About $85 \%$ delivered vaginally, and only $23(7.7 \%)$ initiated breastfeeding within 1 hour after delivery. Approximately half of the sample intended to breastfeed for 6 months or longer, and 79 (26.3\%) had not decided at the time of the first survey. The mean health literacy was 46.9 (standard deviation \pm 3.4 ), the mean breastfeeding knowledge was 6.8 (standard deviation \pm 1.7 ), and the mean breastfeeding self-efficacy was 35.9 (standard deviation \pm 9.7 ) (Table 1).
Table 1. Characteristics of Primiparous WOMEN $(N=300)$

\begin{tabular}{|c|c|}
\hline Variable & $\mathrm{n}(\%)$ \\
\hline \multicolumn{2}{|l|}{ Age (years) } \\
\hline$<25$ & $27(9.0)$ \\
\hline $25-30$ & $121(40.3)$ \\
\hline $31-35$ & $117(39.0)$ \\
\hline$>35$ & 35 (11.7) \\
\hline \multicolumn{2}{|l|}{ Education } \\
\hline High school and below & $80(26.7)$ \\
\hline College/university & $185(61.6)$ \\
\hline Graduate & 35 (11.7) \\
\hline \multicolumn{2}{|l|}{ Employment status } \\
\hline \multicolumn{2}{|l|}{ Before birth } \\
\hline Full-time & $220(73.3)$ \\
\hline No & $80(26.7)$ \\
\hline \multicolumn{2}{|l|}{ At 3 months postpartum } \\
\hline Full-time & $163(60.8)$ \\
\hline No & $105(39.2)$ \\
\hline \multicolumn{2}{|l|}{ Birth method } \\
\hline Cesarean section & $47(15.7)$ \\
\hline Vaginal delivery & $253(84.3)$ \\
\hline \multicolumn{2}{|l|}{ Breastfeeding initiation } \\
\hline$<1$ hour & $23(7.7)$ \\
\hline$\leq 4$ hours & $118(39.3)$ \\
\hline$\leq 24$ hours & $112(37.3)$ \\
\hline$>24$ hours & $47(15.7)$ \\
\hline \multicolumn{2}{|l|}{ Had attended a prenatal classes } \\
\hline Yes & $115(38.5)$ \\
\hline No & $167(55.7)$ \\
\hline \multicolumn{2}{|l|}{ Rooming-in } \\
\hline Yes & $115(38.3)$ \\
\hline No & $185(61.7)$ \\
\hline \multicolumn{2}{|l|}{ Breastfeeding intention } \\
\hline$<6$ months & $54(18)$ \\
\hline 6 months & $116(38.7)$ \\
\hline$>6$ months & $51(17)$ \\
\hline Not sure & $79(26.3)$ \\
\hline Health literacy [mean \pm SD (range)] & $46.9 \pm 3.4(0-50)$ \\
\hline $\begin{array}{l}\text { Breastfeeding knowledge } \\
\quad([\text { mean } \pm \text { SD (range) }]\end{array}$ & $6.8 \pm 1.7(0-10)$ \\
\hline $\begin{array}{l}\text { Breastfeeding self-efficacy } \\
\quad[\text { mean } \pm \text { SD (range) }]\end{array}$ & $35.9 \pm 9.7(12-60)$ \\
\hline
\end{tabular}

$\mathrm{SD}$, standard deviation.

There was a sharp decline in the rate of exclusive breastfeeding in our study sample. The proportion of exclusive breastfeeding was $66 \%(n=198)$ before hospital discharge. It decreased to $37.5 \%(n=104)$ at 1 month postpartum, $30.2 \%$ $(n=81)$ at 3 months postpartum, and $4.7 \%(n=12)$ at 6 months postpartum (Fig. 1).

The factors associated with exclusive breastfeeding inhospital and at 1 and 3 months postpartum are shown in Table 2. Older women were less likely to breastfeed exclusively before discharge. Women living in a metropolitan city had a smaller likelihood of breastfeeding exclusively than those living outside a metropolitan city. Women who had a vaginal delivery, rooming-in practice, early breastfeeding initiation, and positive self-efficacy were more likely to breastfeed exclusively during the hospital stay. Women who reported 
FIG. 1. Percentages of exclusive breastfeeding, mixed breastfeeding, and formula feeding during the initial hospital stay and at 1 month, 3 months, and 6 months postpartum.

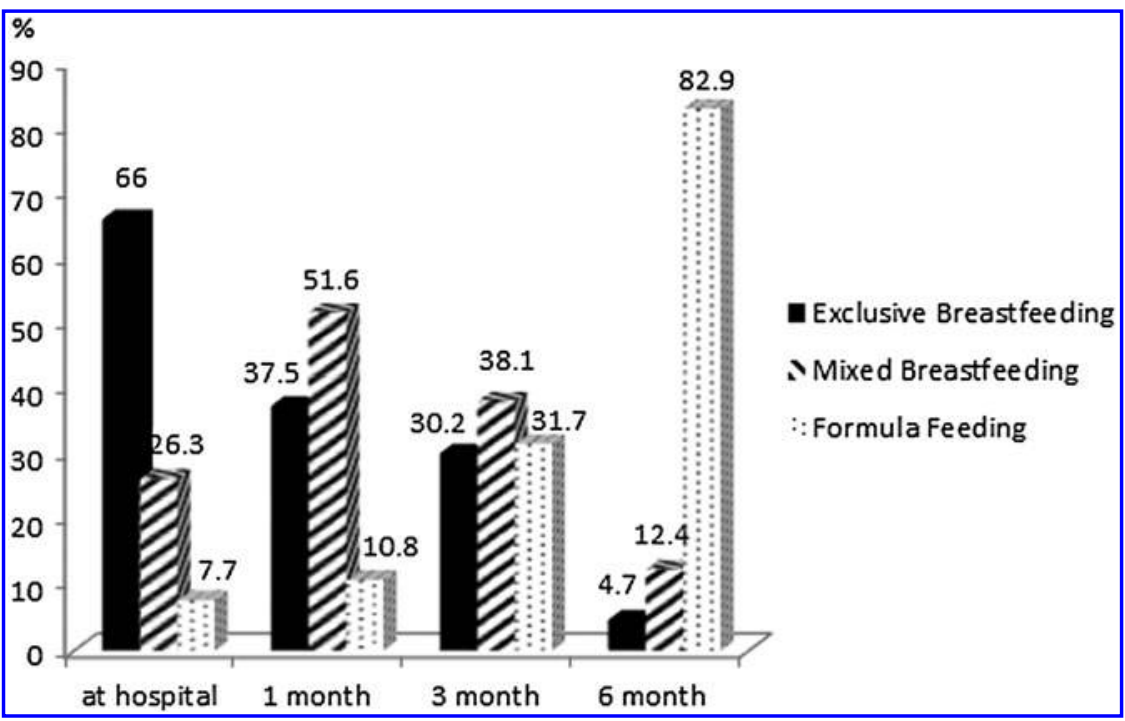

Table 2. Logistic Regression of Factors on Exclusive Breastfeeding at Three Time Points

Odds ratio (95\% CI) for exclusive breastfeeding

\begin{tabular}{|c|c|c|c|}
\hline Variable & Before discharge $(\mathrm{n}=300)$ & At 1 month $(\mathrm{n}=277)$ & At 3 months $(\mathrm{n}=268)$ \\
\hline \multicolumn{4}{|l|}{ Age (years) } \\
\hline$<25$ & Reference & Reference & Reference \\
\hline $25-30$ & $0.28(0.08,1.01)$ & $0.39(0.15,0.99)^{\mathrm{a}}$ & $0.97(0.36,2.60)$ \\
\hline $31-35$ & $0.17(0.05,0.61)^{\mathrm{a}}$ & $0.31(0.12,0.81)^{\mathrm{a}}$ & $1.10(0.41,2.95)$ \\
\hline$>35$ & $0.21(0.05,0.84)^{\mathrm{a}}$ & $0.13(0.04,0.44)^{\mathrm{a}}$ & $0.32(0.08,1.26)$ \\
\hline \multicolumn{4}{|l|}{ Education } \\
\hline High school and below & Reference & Reference & Reference \\
\hline College/university & $0.86(0.49,1.51)$ & $0.77(0.43,1.36)$ & $1.53(0.78,3.01)$ \\
\hline Graduate & $0.77(0.33,1.77)$ & $1.97(0.85,4.60)$ & $6.11(2.41,15.50)^{\mathrm{a}}$ \\
\hline \multicolumn{4}{|l|}{ Residence } \\
\hline Yi-Lan (suburban) & Reference & Reference & Reference \\
\hline Taipei (metropolitan) & $0.38(0.23,0.62)^{\mathrm{a}}$ & $1.05(0.65,1.71)$ & $1.88(1.11,3.19)^{\mathrm{a}}$ \\
\hline Full-time job prenatally & $0.84(0.49,1.46)$ & $0.75(0.43,1.31)$ & - \\
\hline Full-time job at 3 months & - & - & $0.47(0.28,0.80)^{\mathrm{a}}$ \\
\hline \multicolumn{4}{|l|}{ Birth method } \\
\hline Cesarean section & Reference & Reference & Reference \\
\hline Vaginal delivery & $3.20(1.69,6.07)^{\mathrm{a}}$ & $1.24(0.62,2.48)$ & $1.07(0.52,2.18)$ \\
\hline \multicolumn{4}{|l|}{ Breastfeeding initiation } \\
\hline$<1$ hour & $38.82(4.8,314.0)$ & $3.67(1.20,11.17)$ & $3.07(0.94,9.99)$ \\
\hline$\leq 4$ hours & $3.58(1.76,7.26)^{\mathrm{a}}$ & $2.21(0.96,5.08)$ & $1.92(0.76,4.80)$ \\
\hline$\leq 24$ hours & $4.41(2.14,9.09)^{\mathrm{a}}$ & $2.59(1.12,5.95)^{\mathrm{a}}$ & $2.12(0.85,5.31)$ \\
\hline$>24$ hours & Reference & Reference & Reference \\
\hline Had attended prenatal classes & $1.07(0.66,1.75)$ & $1.01(0.61,1.67)$ & $1.34(0.80,2.27)$ \\
\hline Rooming-in & $2.43(1.47,4.04)^{\mathrm{a}}$ & $0.89(0.54,1.45)$ & $0.77(0.46,1.31)$ \\
\hline \multicolumn{4}{|l|}{ Intention } \\
\hline$<6$ months & Reference & Reference & Reference \\
\hline 6 months & $0.67(0.24,1.49)$ & $2.25(1.11,4.57)^{\mathrm{a}}$ & $6.67(2.46,18.13)^{\mathrm{a}}$ \\
\hline$>6$ months & $0.46(0.17,1.21)$ & $3.03(1.33,6.89)^{\mathrm{a}}$ & $8.10(2.74,23.95)^{\mathrm{a}}$ \\
\hline Health literacy & $1.01(0.94,1.08)$ & $1.10(1.01,1.20)^{\mathrm{a}}$ & $1.05(0.95,1.16)$ \\
\hline Breastfeeding knowledge & $1.14(0.99,1.31)$ & $1.33(1.14,1.56)^{\mathrm{a}}$ & $1.17(0.99,1.38)$ \\
\hline Breastfeeding self-efficacy & $1.09(1.06,1.13)^{\mathrm{a}}$ & $1.04(1.02,1.07)^{\mathrm{a}}$ & $1.03(1.01,1.06)^{\mathrm{a}}$ \\
\hline
\end{tabular}

\footnotetext{
${ }^{\mathrm{a}}$ Significant difference.
}

$\mathrm{CI}$, confidence interval. 
exclusive breastfeeding at 1 month postpartum tended to be younger and had earlier initiation of breastfeeding, longer intention of breastfeeding, a higher level of health literacy, better breastfeeding knowledge, and greater breastfeeding self-efficacy. At 3 months of postpartum, women with a graduate degree, living in a metropolitan city, intending to breastfeeding 6 months or longer, and having greater selfefficacy had a significantly higher likelihood of breastfeeding exclusively. Women with a full-time job were less likely to practice exclusive breastfeeding.

We used the WHO's 6-month breastfeeding recommendation as a reference to examine maternal and hospital factors that predicted early cessation of breastfeeding within 6 months of birth. Table 3 displays the results of Cox regression modeling. Model 1 includes maternal and hospital factors and shows that women who intended to breastfeed less than 6 months had $42 \%$ greater risk of early cessation compared with women who intended to breastfeed more than 6 months and that initiating breastfeeding after 24 hours of birth increased the risk of early cessation by $93 \%$ compared with breastfeeding initiation within 1 hour. Adding sociodemographic variables in Model 2 changes the results, which show that breastfeeding self-efficacy and late breastfeeding initiation were negatively related to early cessation of breastfeeding.

Table 3. Predictors of Early Cessation OF BREASTFEEDING $(N=258)$

\begin{tabular}{|c|c|c|}
\hline \multirow[b]{2}{*}{ Variable } & \multicolumn{2}{|c|}{ Hazard ratio $(95 \%$ CI) } \\
\hline & Model 1 & Model 2 \\
\hline Health literacy & $0.99(0.94,1.06)$ & $1.01(0.94,1.08)$ \\
\hline $\begin{array}{l}\text { Breastfeeding } \\
\text { knowledge }\end{array}$ & $0.93(0.82,1.04)$ & $0.95(0.84,1.07)$ \\
\hline $\begin{array}{l}\text { Breastfeeding } \\
\text { self-efficacy }\end{array}$ & $0.99(0.97,1.01)$ & $0.98(0.96,0.99)^{\mathrm{a}}$ \\
\hline \multicolumn{3}{|c|}{ Breastfeeding intention versus $>6$ months } \\
\hline$<6$ months & $1.42(1.13,1.68)^{\mathrm{a}}$ & $1.08(0.92,1.16)$ \\
\hline 6 months & $1.01(0.91,1.08)$ & $0.93(0.83,1.09)$ \\
\hline Cesarean section & $1.24(0.76,2.12)$ & $1.35(0.78,2.33)$ \\
\hline Rooming-in & $0.98(0.69,1.39)$ & $1.13(0.77,1.65)$ \\
\hline $\begin{array}{l}\text { Not attending } \\
\text { prenatal classes }\end{array}$ & $1.07(0.75,1.52)$ & $1.13(0.77,1.65)$ \\
\hline \multicolumn{3}{|c|}{ Breastfeeding initiation versus $<1$ hour } \\
\hline$\leq 4$ hours & $1.40(0.71,1.73)$ & $1.53(1.05,1.97)^{\mathrm{a}}$ \\
\hline$\leq 24$ hours & $1.56(0.79,1.81)$ & $1.32(0.82,2.19)$ \\
\hline$>24$ hours & $1.93(1.11,4.36)^{\mathrm{a}}$ & $1.67(0.98,2.42)$ \\
\hline \multicolumn{3}{|c|}{ Age versus $>35$ years } \\
\hline$<25$ years & & $0.36(0.17,0.73)^{\mathrm{a}}$ \\
\hline $25-30$ years & & $0.73(0.45,1.15)$ \\
\hline $31-35$ years & & $0.82(0.54,1.28)$ \\
\hline \multicolumn{3}{|c|}{ Education graduate versus } \\
\hline $\begin{array}{l}\text { High school } \\
\text { and below }\end{array}$ & & $2.87(1.10,7.24)$ \\
\hline College/university & & $2.07(1.04,5.40)$ \\
\hline $\begin{array}{l}\text { Yi-Lan County } \\
\text { residence }\end{array}$ & & $0.83(0.54,1.28)$ \\
\hline $\begin{array}{l}\text { Full-time job at } \\
3 \text { months } \\
\text { postpartum }\end{array}$ & & $1.28(0.88,1.85)$ \\
\hline
\end{tabular}

${ }^{\text {a }}$ Significant difference.

CI, confidence interval.

\section{Discussion}

The present study showed that although all women in the study sample initiated breastfeeding during the hospital stay, fewer than $8 \%$ initiated breastfeeding within an hour following the birth and $34 \%$ had already given up exclusively breastfeeding before leaving the hospital. We found a significant decrease in exclusive breastfeeding during the 6 months postpartum, with women practicing any form of breastfeeding at 6 months postpartum constituting a minority of the study sample (14.7\%). These findings are consistent with prior studies in the United States, Australia, China, and Hong Kong that found a precipitous discontinuation of breastfeeding after hospital discharge..$^{9,26-28}$

The present study confirms and extends previous study results that determinants of breastfeeding were multifaceted. ${ }^{8,9,27,29}$ Consistent with previous research, ${ }^{10,12,14}$ we found that two BFHI practices (i.e., initiation of breastfeeding within 1 hour and rooming-in practice) contributed to enhancing successful breastfeeding. However, a small proportion of women in this study was compliant with these two BFHI recommendations. In a nationwide survey in Taiwan, $54.9 \%$ of women reported early skin-to-skin contact, and $33.8 \%$ had rooming-in practice. ${ }^{30}$ The poor compliance may be due to maternity routines and medical staff members' attitude and support. ${ }^{15}$ Although the two hospitals where the study was conducted were both BFHI accredited, the accreditation requires only early skin-to-skin contact, and there is no requirement regarding breastfeeding initiation. Furthermore, the standards are so low (10-20 minutes of skinin-skin contact within 30 minutes of delivery) that it is easy for hospitals to meet the requirement without leading to any significant impact on the timing of breastfeeding initiation.

The cultural practice of postnatal confinement among Taiwanese women may also play a role in influence roomingin practice. ${ }^{30}$ In Chinese culture, new mothers are expected rest as much as possible after birth to restore their health. Rooming-in practice may interrupt their rest. This explanation is consistent with our finding that a significantly higher proportion of women in the younger age group adhered to rooming-in practice, as younger women may be less inclined to observe the traditional custom.

Our analysis showed that the influence of hospital interventions (i.e., rooming-in practice) on exclusive breastfeeding was not sustainable after the hospital discharge. Maternal psychosocial factors, such as intention, health literacy, and selfefficacy, were more important determinants for breastfeeding exclusivity once women were discharged from the hospital. In Taiwan, the national health insurance program covers 3 days of hospital stay for a vaginal delivery and 5 days for a cesarean section. Except for an outpatient follow-up at 6 weeks postpartum, community-based postpartum care is generally unavailable. It has been reported that the 2 weeks after the delivery are a critical period of premature discontinuation of breastfeeding because this is when various breastfeeding difficulties seem to arise and intensify. ${ }^{8,31}$ In a qualitative study of breastfeeding barriers, ${ }^{32}$ the majority of women experienced at least one barrier to breastfeeding during the early postpartum period.

Once discharged from the hospital and without the support and consultation of hospital staff, first-time mothers may face a range of breastfeeding difficulties, such as breastfeeding-related discomfort and inconvenience, inadequate production of milk, 
and social pressures against breastfeeding. ${ }^{6,33}$ These problems are difficult to overcome unless those first-time mothers have sufficient knowledge and adequate health literacy to acquire useful information and skills in the early postpartum period. In addition to learned skills, practice, and support, productive breastfeeding requires perseverance and successful early breastfeeding experience, ${ }^{6}$ hence our findings of a positive relationship between self-efficacy and exclusive breastfeeding in the postpartum period. These findings suggest that maternity health providers need to provide new mothers, while they are still in the hospital, guidance on how to prevent or manage common breastfeeding difficulties. ${ }^{8}$ New mothers with low health literacy may need additional assistance to manage breastfeeding difficulties.

This study provides evidence that breastfeeding intention and self-efficacy are important predictors of breastfeeding cessation within the first 6 months of delivery, corroborating the findings of some previous studies. ${ }^{29,31}$ Furthermore, our findings reveal a substantial gap between exclusive breastfeeding intention and exclusive breastfeeding duration. More than $55 \%$ of first-time mothers in our sample wished to breastfeed their children for the recommended 6 months or longer; however, only $17.1 \%$ of study participants continued with some form of breastfeeding at 6 months postpartum. Research suggests that many women decide how they would feed their baby before they are pregnant or early on during pregnancy. ${ }^{34}$ A women's decision to breastfeed may differ from those that are associated with a woman's ability to establish exclusive breastfeeding successfully. ${ }^{8}$ Further studies should identify how a woman's intention changes over time. Given the importance of breastfeeding self-efficacy and intention, interventions should use multifaceted strategies to encourage women to decide on breastfeeding by clearly communicating the benefits of breastfeeding, enhancing their breastfeeding skills, strengthening their self-efficacy prior to and during pregnancy, and reinforcing the intention across the perinatal continuum.

That said, it is interesting to note that no significant relationship was observed between prenatal classes and breastfeeding exclusivity and duration. It is possible that breastfeeding did not receive sufficient coverage in birth preparation classes in Taiwan. Another plausible explanation is that prenatal classes tend to focus on knowledge-based information, which is inadequate for encouraging and sustaining successful breastfeeding. To the extent these explanations are valid, prenatal classes need to provide breastfeeding skills and build positive social norms in order to increase exclusive breastfeeding intention and duration. ${ }^{7,34,35}$

It is not surprising to find in our analysis that returning to work was a significant barrier to exclusive breastfeeding, which was revealed in many previous studies. ${ }^{5,35-37}$ Lack of support from workplace or unfavorable working conditions may compel working mothers to consider early termination of breastfeeding exclusivity and introduction of formula during or immediately after the maternity leave. ${ }^{38,39}$ Policy initiatives and resources to promote work environments conducive to breastfeeding may effectively increase the rate of exclusive breastfeeding among working mothers.

Our findings must be interpreted in consideration of the study weaknesses. First, we collected data on women's cognitive and psychological characteristics at the baseline survey. The data did not capture any change in first-time mothers' health literacy competence and psychological characteristics in the postpartum period. Future studies may assess variation in maternal cognitive and psychological conditions along with the change in breastfeeding practice over time. This will broaden our understanding of how maternal health literacy, intention, and self-efficacy change over time and their impact on breastfeeding behaviors. Second, our study sample was hospital based, with participants recruited purposefully at two hospitals. Study participants may not be representative of all primiparous women during the study period, thus limiting the generality of our findings. Third, the small sample size may limit the statistical power in our analysis, and our results may be biased towards the null. Fourth, although we found no demographic differences between women who remained in the study and women who were lost to follow-up, we could not exclude the possibility of attrition bias in this study. Finally, our measures of breastfeeding exclusivity and duration were based on self-reports, and we therefore could not rule out social desirability bias. A recent nationwide survey on breastfeeding rate in Taiwan reported similar rates, ${ }^{5}$ suggesting that the breastfeeding data collected in our sample were reliable.

\section{Conclusions}

The current study adds insights into breastfeeding determinants across different postpartum periods and suggests important windows of opportunity for breastfeeding interventions and management. In this study, early breastfeeding initiation and rooming-in practice were strong predictors of exclusive breastfeeding during the hospital stay. However, the rates of early breastfeeding initiation and rooming-in practice were low in comparison with those of BFHI-accredited hospitals in other countries, likely to due to traditional maternal beliefs and practices in Taiwanese culture. To the extent this is true, interventions that are aimed to increase early breastfeeding initiation should be designed with such cultural considerations and be tailored to the cultural beliefs and practices of women and their family. Our findings also suggest that interventions to increase breastfeeding exclusivity and duration of first-time mothers should focus on maternal health literacy, breastfeeding intention, and self-efficacy enhancement during their pregnancy and after the delivery.

\section{Acknowledgments}

This study was funded by grant 98A-C-D165 from the Ministry of Education, Aim for the Top University Plan, Taiwan. We would like to thank all the women who participated in the study. We thank the research team for their assistance and two hospitals for their collaboration during the conducting of this research.

\section{Disclosure Statement}

No competing financial interests exist.

\section{References}

1. Ip S, Chung M, Raman G, et al. A summary of the Agency for Healthcare Research and Quality's evidence report on breastfeeding in developed countries. Breastfeed Med 2009; 4(Suppl 1):S17-S30.

2. Oddy WH, Kendall GE, Li J, et al. The long-term effects of breastfeeding on child and adolescent mental health: A 
pregnancy cohort study followed for 14 years. 2010;156:568-574.

3. World Health Organization. Infant and Young Child FeedingModel Chapter for Textbooks. World Health Organization, Geneva, 2009.

4. Data and Analytics Section, Division of Data, Research and Policy, UNICEF. Infant and Young Child Feeding. 2014. Available at http://data.unicef.org/nutrition/iycf (accessed April 7, 2015).

5. Chuang C-H, Chang P-J, Chen Y-C, et al. Maternal return to work and breastfeeding: A population-based cohort study. Int J Nurs Stud 2010;47:461-474.

6. Avery A, Zimmermann K, Underwood PW, et al. Confident commitment is a key factor for sustained breastfeeding. Birth 2009;36:141-148.

7. de Jager E, Broadbent J, Fuller-Tyszkiewicz M, et al. The role of psychosocial factors in exclusive breastfeeding to six months postpartum. Midwifery 2014;30:657-666.

8. Scott J, Binns C, Oddy W, et al. Predictors of breastfeeding duration: evidence from a cohort study. Pediatrics 2006; 117:e646-e655.

9. O'Brien M, Buikstra E, Hegney D. The influence of psychological factors on breastfeeding duration. J Adv Nurs 2008;63:397-408.

10. Chien L-Y, Tai C-J, Ko Y-L, et al. The number of Baby Friendly Hospital practices experienced by mothers is positively associated with breastfeeding: A questionnaire survey. Int J Nurs Stud 2007;44:1138-1146.

11. Forster DA, McLachlan HL. Breastfeeding initiation and birth setting practices: A review of the literature. $\underline{J \text { Mid- }}$ wifery Womens Health 2007;52:273-280.

12. Tarrant M, Wu KM, Fong DYT, et al. Impact of BabyFriendly Hospital practices on breastfeeding in Hong Kong. Birth 2011;38:238-245.

13. Merten S, Fratva J, Ackermann-Liebrich U. Do BabyFriendly Hospitals influence breastfeeding duration on a national level? Pediatrics 2005;116:e702-e708.

14. Perrine CG, Scanlon KS, Li R, et al. Baby-Friendly Hospital practices and meeting exclusive breastfeeding intention. Pediatrics 2012;130:54-60.

15. Semenic S, Loiselle C, Gottlieb L. Predictors of the duration of exclusive breastfeeding among first-time mothers. Res Nurs Health 2008;31:428-441.

16. Glassman ME, McKearney K, Saslaw M, et al. Impact of breastfeeding self-efficacy and sociocultural factors on early breastfeeding in an urban, predominantly Dominican community. Breastfeed Med 2014;9:301-307.

17. Lee S-YD, Tsai TI, Tsai YW, Kuo KN. Health literacy and women's health-related behaviors in Taiwan. Health Educ Behav 2012;39:210-218.

18. Nielsen-Bohlman L, Panzer AM, Kindig DA. Health Literacy: A Prescription to End Confusion. Institute of Medicine, Washington, DC, 2004.

19. Osborn CY, Paasche-Orlow MK, Bailey SC, et al. The mechanisms linking health literacy to behavior and health status. Am J Health Behav 2011;35:118-128.

20. von Wagner C, Steptoe A, Wolf MS, et al. Health literacy and health actions: A review and a framework from health psychology. Health Educ Behav 2009;36:860-877.

21. Kaufman H, Skipper K, Small L, et al. Effect of literacy on breastfeeding outcomes. South Med J 2001;94:293-296.

22. Fein SB, Labiner-Wolfe J, Shealy KR, et al. Infant Feeding Practices Study II: Study methods. Pediatrics 2008; 122(Suppl 2):S2-S35.
23. Labbok M, Krasovec K. Toward consistency in breastfeeding definitions. Stud Fam Plan 1990;21:226-230.

24. Tsai TI, Lee SY, Tsai YW, et al. Methodology and validation of health literacy scale development in Taiwan. J Health Commun 2011;16:50-61.

25. Dai X, Dennis C. Translation and validation of the Breastfeeding Self-Efficacy Scale into Chinese. I Midwifery Womens Health 2003;48:350-356.

26. Jiang H, Li M, Yang D, et al. Awareness, intention, and needs regarding breastfeeding: Findings from first-time mothers in Shanghai, China. Breastfeed Med 2012;7:526-534.

27. Jones J, Kogan MD, Singh GK, et al. Factors associated with exclusive breastfeeding in the United States. Pediatrics 2011;128:1117-1125.

28. Tarrant M, Fong DYT, Wu KM, et al. Breastfeeding and wean practices among Hong Kong mothers: A prospective study. BMC Pregnancy Childbirth 2011;10:1-12.

29. Forster D, McLachlan H, Lumley J. Factors associated with breastfeeding at six months postpartum in a group of Australian women. Int Breastfeed J 2006;1:18.

30. Chiou S-T, Chen L-C, Yeh H, et al. Early skin-to-skin contact, roomin-in, and breastfeeding: A comparison of the 2004 and 2011 National Surveys in Taiwan. Birth 2014;41:33-38.

31. DiGirolamo D, Thompson $\mathrm{N}$, Martorell $\mathrm{R}$, et al. Intention or experience? Predictors of continued breastfeeding. Health Educ Behav 2005;32:208-226.

32. Teich AS, Barnett J, Bonuck K. Women's perceptions of breastfeeding barriers in early postpartum period: A qualitative analysis nested in two randomized controlled trials Breastfeed Med 2014;9:9-15.

33. Santo LCdE, De Oliveira LD, Giugliani ERJ. Factors associated with low incidence of exclusive breastfeeding for the first 6 months. Birth 2007;34:212-219.

34. Newby R, Brodribb W, Ware RS, et al. Infant feeding knowledge, attitudes, and beliefs predict antenatal intention among first-time mothers in Queensland. Breastfeed Med 2014;9:266-272.

35. Ishak S, Adzan NAM, Quan LK, et al. Knowledge and beliefs about breastfeeding are not determinants for successful breastfeeding. Breastfeed Med 2014;9:308-312.

36. Idris NS, Sastroasmoro S, Hidayati F, et al. Exclusive breastfeeding plan of pregnant Southeast Asian women: What encourages them? Breastfeed Med 2013;8:317-320.

37. Lee WTK, Wong E, Lui SSH, et al. Decision to breastfeed and early cessation of breastfeeding in infants below 6 months old-A population-based study of 3204 infants in Hong Kong. Asia Pac J Clin Nutr 2007;16:163-171.

38. Dyson L, Renfrew MJ, McFadden A, et al. Policy and public health recommendations to promote the initiation and duration of breast-feeding in developed country settings. Public Health Nutr 2009;13:137-144.

39. Lawrence RA. Breastfeeding triumphs. Birth 2012;39: 311-314.

Address correspondence to:

Tzu-I Tsai, PhD

School of Nursing

National Yang-Ming University

Room 307, Nursing Building 155, Sec. 2, Linong Street

Beitou District, 112

Taipei, Taiwan

E-mail: titsai@ym.edu.tw 
This article has been cited by:

1. Maya Bunik. 2017. The Pediatrician's Role in Encouraging Exclusive Breastfeeding. Pediatrics in Review 38:8, 353-368. [Crossref]

2. Aifric O'Sullivan, Marie Farver, Jennifer T. Smilowitz. 2015. Article Commentary: The Influence of Early Infant-Feeding Practices on the Intestinal Microbiome and Body Composition in Infants. Nutrition and Metabolic Insights 8s1, NMI.S29530. [Crossref] 